\title{
Qualità della vita misurata attraverso il Kidney Disease Quality of Life-Short Form (KDQOL-SF): differenze in relazione al trattamento dialitico
}

\author{
M.N. Rodi, C. Mura
}

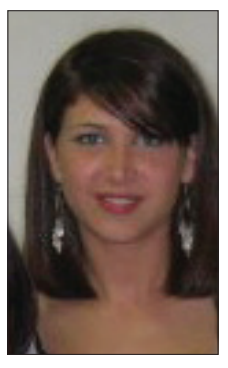

Maria Novella Rodi

\section{Introduzione}

In Italia 153 persone per milione di popolazione, secondo i dati dell'Italian Register of Dialysis and Transplantation (RIDT), sopravvivono grazie alla dialisi.

La convivenza con una patologia cronica si traduce sempre per i pazienti in una "esperienza di malattia" che, oltre agli aspetti organici, coinvolge anche dimensioni emotive e psicosociali.

La valutazione personale del paziente circa la propria condizione in relazione alle variabili strutturali e psicologiche è stata solo recentemente considerata come indicatore valido della qualità della vita $(\mathrm{QdV}) \mathrm{e}$ come elemento di giudizio rilevante in merito alla valutazione e alla scelta tra i diversi trattamenti dialitici oggi disponibili (1).

Il New England Journal of Medicine nel 1985 pubblicò un lavoro di Evans sulla qualità della vita dei pazienti dializzati e trapiantati, confrontandoli con la popolazione generale, e attirò così, forse per la prima volta, l'attenzione della Comunità Scientifica Nefrologica sui problemi della riabilitazione e dell'adattamento di questi pazienti. In tale lavoro, furono evidenziate le notevoli limitazioni che la dialisi e, ai tempi, anche il trapianto, creavano nella vita del paziente sia in campo lavorativo che nelle attività quotidiane (2).

Da una rassegna della letteratura, emergono alcuni fattori che correlano positivamente con la $\mathrm{QdV}$ come trapianto, esercizio fisico, lavoro, supporto sociale e altri negativamente, quali comorbilità, depressione, perdita di autonomia, dipendenza dalla macchina e molti altri.
La valutazione della qualità del trattamento dialitico percepita dal paziente non è ancora una procedura di routine nella maggior parte delle istituzioni. Rispetto a una valutazione attenta degli indicatori di performance riguardanti la depurazione, infatti, poca enfasi è riposta sulla valutazione oggettiva della percezione del paziente, in maniera più o meno indipendente dai livelli di cura prestati (3).

A fronte di una eccellente gestione clinica, non sempre ci si chiede se il paziente sia soddisfatto o meno del trattamento ricevuto. Valutare questo aspetto sarebbe essenziale per mettere in atto degli interventi volti al miglioramento della $\mathrm{QdV}$ dei pazienti stessi. Una presa in carico del paziente che tenga in considerazione la sua realtà complessiva, fatta anche di bisogni e problematiche socio-ambientali, familiari o psicologiche, se effettuata correttamente, potrebbe migliorare profondamente la sua condizione, restituendogli energie e motivazione per affrontare meglio il trattamento dialitico.

Comincia a farsi strada la convinzione che anche nelle fasi precedenti il trattamento si debba lavorare nello sviluppo della consapevolezza riguardo le scelte o le opportunità terapeutiche.

Sarebbe quindi opportuno spiegare ai pazienti durante la predialisi i vantaggi e gli svantaggi delle diverse metodiche dialitiche rispetto alla qualità della vita, in modo che la scelta del trattamento non derivi unicamente dal personale medico, ma che loro stessi siano considerati parte attiva di tutto il processo decisionale. Se i pazienti parteciperanno più attivamente alle decisioni inerenti il proprio trattamento, si percepiranno meno come "malati" e riusciranno a conservare meglio la propria quotidianità e le consuete attività lavorative e di svago. 


\section{Materiali e Metodi}

L'obiettivo della ricerca è quello di verificare se esistano differenze nella QdV in base al trattamento svolto (emodialisi e dialisi peritoneale). La ricerca ha coinvolto 195 pazienti (157 in emodialisi e 38 in peritoneale), afferenti a 5 Centri nefrologici della Toscana: Arezzo, Firenze OSMA, Montevarchi, Pistoia, Siena.

Lo strumento d'indagine è stato un questionario self-report, composto da 2 sezioni, la prima riguardante i dati anagrafici e la seconda la qualità della vita attraverso il Kidney Disease Quality of Life-Short Form (KDQOLSF) (4). I dati raccolti sono stati esaminati attraverso analisi descrittive, correlazioni e analisi della varianza, dopo aver estratto casualmente un campione omogeneo per età, genere e condizione clinica, composto da 31 pazienti in emodialisi e 31 in peritoneale.

\section{Risultati}

Dalle analisi quantitative eseguite, risultano alcune differenze nei due tipi di trattamento esaminati.

In Dialisi Peritoneale i valori medi delle scale variano da un minimo di 31,5 punti nel "Ruolo e salute fisica" a un massimo di 83,5 nella scala "Vicinanza dello staff".

In Emodialisi, il range va da 41,3 punti nella scala "Salute generale” a 88,3 nella scala "Vicinanza dello staff” (Tab. I). I soggetti sottoposti a dialisi peritoneale hanno punteggi medi superiori agli emodializzati nella scala dell'attività fisica, in quella della salute in generale e nella salute mentale (Tab. I).
L'analisi della varianza a una via, eseguita tra i peritoneali e gli emodializzati (Tab. II), rileva solamente tre differenze statisticamente significative, con medie superiori nel sottogruppo emodalisi nelle seguenti scale: attività sociali, funzioni sessuali e incoraggiamento dello staff.

\section{Discussione}

Dai risultati emergono differenze significative tra i due sottogruppi, maggiori negli emodializzati, in relazione alle funzioni sessuali. Sembrerebbe dunque che i soggetti sottoposti ad emodialisi abbiano minori problemi sia nel provare piaceri sessuali che nella prima fase dell'eccitamento.

Questo risultato è in accordo con l'indagine eseguita dal CENSIS, dove emerge che i peritoneali, in seguito alla presenza permanente del catetere nella cavità addominale, unito alla sensazione di una minore attraenza fisica, lamentino forti problemi nella vita sessuale (5).

Un'altra differenza significativa emerge nella scala "Incoraggiamento dallo staff" e "Attività sociali". Sembrerebbe che gli emodializzati ottengano un superiore incoraggiamento da parte del personale della dialisi ed esercitino le normali attività sociali senza interferenze dovute ai problemi fisici o emotivi, rispetto ai peritoneali. Questo può essere confermato dal fatto che i pazienti sottoposti a dialisi extracorporea, stando a contatto con lo staff medico e infermieristico per tempi più lunghi rispetto ai peritoneali, ricevono da questo un più alto incoraggiamento verso l'autonomia e l'indipendenza, sia per quanto concerne il trattamento che la malattia renale

TABELLA I - LE SCALE DEL KDQOL-SF IN BASE AL DIVERSO TIPO DI TRATTAMENTO

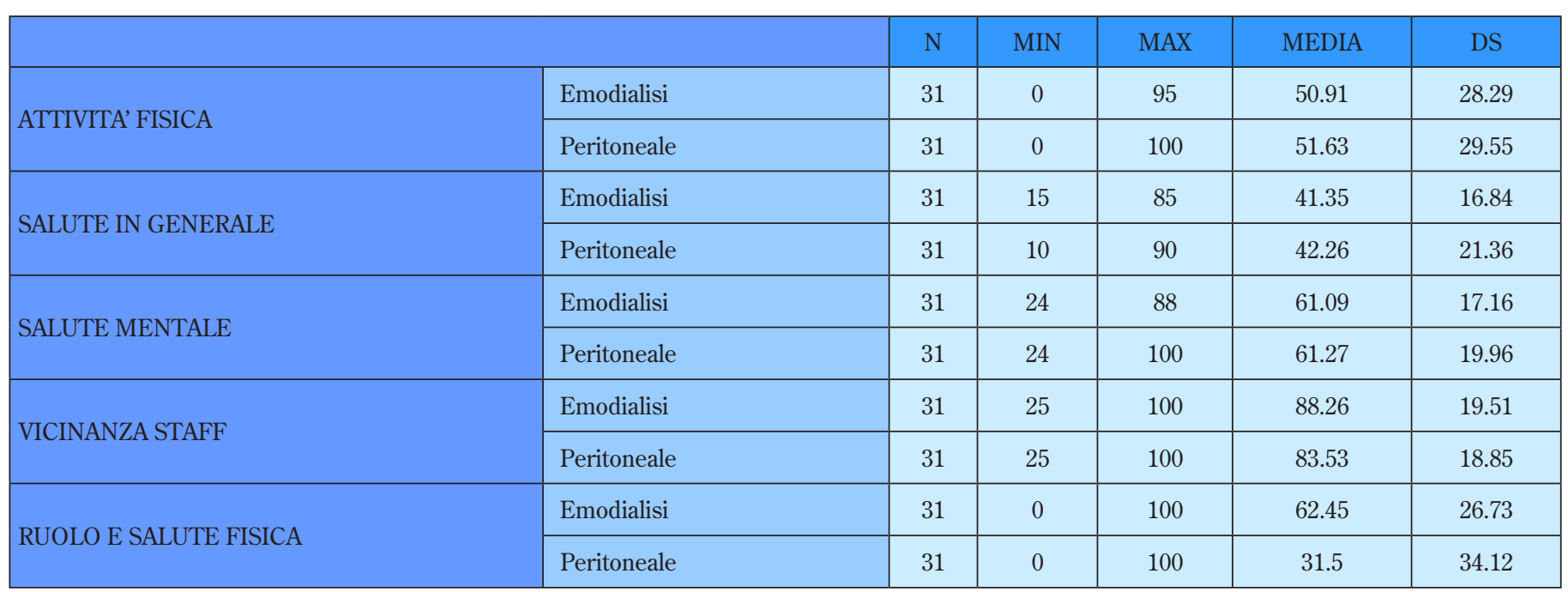


TABELLA II - RISULTATI SIGNIFICATIVI (P $\approx 0)$ DELL'ANOVA A UNA VIA (F DI FISCHER) NELLE SCALE DEL KDQOL-SF

\begin{tabular}{|l|c|c|}
\hline & F & $P$ \\
\hline Attività sociali & 13.99 & 0 \\
\hline Funzioni sessuali & 7.99 & 0.006 \\
\hline Incoraggiamento Staff & 12.4 & 0.007 \\
\hline
\end{tabular}

Variabile Indipendente ANOVA = Tipologia di trattamento.

nel suo complesso. Ciò si traduce in minori interferenze emotive e fisiche sulle attività sociali che il soggetto svolge quotidianamente.

Emergerebbe che il paziente peritoneale, per il fatto che non ha un rapporto altrettanto forte con lo staff medico e infermieristico, perché la DP, essendo una tecnica domiciliare non prevede sedute in reparto ma solo periodici controlli di routine, percepisca un minore incoraggiamento. Inoltre, esaminando le scale del KDQOL-SF, si nota una correlazione positiva, negli emodializzati, tra la scala "Soddisfazione del paziente riguardo all'assistenza ricevuta" e quella "Incoraggiamento da parte dello staff medico e paramedico".

Si potrebbe dedurre che l'incoraggiamento ricevuto dal paziente da parte del personale del Centro dialisi per quanto concerne l'indipendenza e l'aiuto ad affrontare la terapia e la malattia renale in genere, faccia modificare la valutazione dell'assistenza ricevuta.

Questo potrebbe dimostrare che nella terapia emodialitica, in cui è presente un maggior contatto con il Centro e il personale rispetto alla terapia peritoneale, si percepisca maggiormente la vicinanza dello staff, elemento questo che farebbe aumentare sia la soddisfazione per le cure ricevute sia la compliance e l'aderenza al trattamento (6).

Inoltre, sempre nei soggetti in emodialisi, lo stato lavorativo correla positivamente con il supporto sociale, il che è in linea con le altre ricerche esaminate: riuscire a mantenere, seppur nelle difficoltà, una vita professionale attiva comporterebbe un aumento del supporto sociale percepito, fattore quest'ultimo di protezione per gli stati depressivi e ansiosi, dal momento che il sentirsi ancora utili dal punto di vista lavorativo, soprattutto nell'emodialisi, terapia più invalidante, fa sì che il paziente non si percepisca solo come "malato", ma come un "individuo" che ha ancora il suo posto nel mondo e il suo ruolo di utilità sociale.

\section{Conclusioni}

Dai risultati della ricerca si evince che la dipendenza dal personale sanitario e dal Centro dialisi in genere, da una lato rappresenterebbe una fonte di frustrazione e di dipendenza che interferisce con le normali attività, dall'altro potrebbe essere considerato un fattore di protezione e di stimolo per il paziente stesso. Egli, frequentando prevalentemente il Centro dialisi, non si sente solo nella sua condizione di malato, ma parte integrante di una "nuova famiglia" che lo aiuta ad affrontare e, in alcuni casi a superare, le limitazioni che l'insufficienza renale comporta. D'altro canto come testimoniano le ultime ricerche della Società Italiana di $\mathrm{Ne}$ frologia, la dialisi peritoneale complessivamente è in calo anche nelle regioni in cui la percentuale di pazienti è più elevata (Nord-Ovest). Inoltre, vista la crescente percentuale di pazienti con elevata comorbilità sembra sempre più difficile proporre una metodica che, essendo domiciliare, è in gran parte autogestita e presuppone una larga rete familiare.

Sarebbe opportuno approfondire l'opportunità di modificare alcuni aspetti socio assistenziali che possano favorire il trattamento di dialisi peritoneale nei pazienti affetti da insufficienza renale cronica.

\section{Riassunto}

Nel 1985 il New England Journal of Medicine pubblicò un rapporto relativo alla Qualità della vita $(\mathrm{QdV})$ nei pazienti dializzati: forse per la prima volta si poneva l'attenzione alla sfera più intima di chi doveva convivere con una patologia cronica. Recentemente gli studi deputati a cogliere i principali aspetti emotivi e psicologici di chi vive una reale "esperienza di malattia", hanno assunto nuova consistenza, scoprendo importanti fattori che influenzano il paziente fin dalla prima fase di pre-dialisi. Nonostante i progressi fatti, manca ancora una routine di valutazione della $\mathrm{QdV}$ che, se strutturata, potrebbe diventare un valore aggiunto per il paziente; spesso a una eccellente gestione clinica non si accompagna, infatti, un'attenta analisi del grado di soddisfazione del malato. L'obiettivo della ricerca è capire se esistano differenze in ambito di QdV tra i pazienti che sono sottoposti a emodialisi rispetto a quelli in dialisi peritoneale. Alla luce dei risultati si evincono aspetti importanti: fattori come la sessualità, l'attività sociale, percezione della vicinanza dello staff, hanno punteggi migliori negli emodializzati. La conclusione è che la normale frustrazione legata all'essere "dipendenti" dal reparto e dal suo staff medico venga ben compensata dal senso di protezione e dai legami umani che li si creano.

Parole chiave: Pre-dialisi, QdV, Emodialisi, Dialisi Peritoneale 
Indirizzo degli Autori:

Maria Novella Rodi, MD

Via Sabotino 5/b

52088 Terranuova

Bracciolini

Arezzo

marianovellarodi@alice.it

\section{Bibliografia}

1. Mingardi G, Apolone G. Misurare la Qualità della Vita correlata alla Salute (QdV-S) nel paziente uremico: una rewiew dei concetti, dei metodi, degli strumenti disponibili e dei risultati. G Ital Nefrol 2005; 22: 477-89.
2. Evans R, Manninen D, GarrisonJ. The quality of life of patients with end-stage renal disease. New Engl J Med 1985; 312: 553-9.

3. Pansini F, Gargano L, Sambati M, Dambrosio N. Valutazione del grado di soddisfazione da trattamento nel paziente in emodialisi: metodologia di ricerca, strumenti, limiti e studio pilota trasversale. G Ital Nefrol 2007; 24 (6): 584-94.

4. Hays R, Kallich J, Mapes D. Kidney disease quality of life short form (KDQOL-SF), Version 1,3: A manual for use and scoring. Santa Monica, CA: RAND; 1997.

5. Censis. Verso l'autosufficienza. Dialisi peritoneale e qualità della vita. Milano: Franco Angeli, 2007; 11-29, 59-83.

6. Kovac J, Patel S, Peterson R, Kimmel P. Aspect of quality of life in hemodialysis patient. Am J Kidney Dis 2002; 39: 1236-44. 